Biol. Cybern. 56, 69-87 (1987)

Biological Cybernetics

(C) Springer-Verlag 1987

\title{
Dynamic Response Properties of Movement Detectors: Theoretical Analysis and Electrophysiological Investigation in the Visual System of the Fly
}

\author{
M. Egelhaaf and W. Reichardt \\ Max-Planck-Institut für biologische Kybernetik, Spemannstrasse 38, D-7400 Tübingen, Federal Republic of Germany
}

\begin{abstract}
Dynamic aspects of the computation of visual motion information are analysed both theoretically and experimentally. The theoretical analysis is based on the type of movement detector which has been proposed to be realized in the visual system of insects (e.g. Hassenstein and Reichardt 1956; Reichardt 1957, 1961; Buchner 1984), but also of man (e.g. van Doorn and Koenderink 1982a, b; van Santen and Sperling 1984; Wilson 1985). The output of both a single movement detector and a one-dimensional array of detectors is formulated mathematically as a function of time. The resulting movement detector theory can be applied to a much wider range of moving stimuli than has been possible on the basis of previous formulations of the detector output. These stimuli comprise one-dimensional "smooth" detector input functions, i.e. functions which can be expanded into a time-dependent convergent Taylor series for any value of the spatial coordinate.

The movement detector response can be represented by a power series. Each term of this series consists of one exclusively time-dependent component and of another component that depends, in addition, on the properties of the pattern. Even the exclusively time-dependent components of the movement detector output are not solely determined by the stimulus velocity. They rather depend in a non-linear way on the weighted sum of the instantaneous velocity and all its higher order time derivatives. The latter point represents another reason - not discussed so far in the literature that movement detectors of the type analysed here do not represent pure velocity sensors.

The significance of this movement detector theory is established for the visual system of the fly. This is done by comparing the spatially integrated movement detector response with the functional properties of the directionally-selective motion-sensitive Horizontal Cells of the third visual ganglion of the fly's brain.
\end{abstract}

These integrate local motion information over large parts of the visual field. The time course of the spatially integrated movement detector response is about proportional to the velocity of the stimulus pattern only as long as the pattern velocity and its time derivatives are sufficiently small. For large velocities and velocity changes of the stimulus pattern characteristic deviations of the response profiles from being proportional to pattern velocity are predicted on the basis of the detector theory developed here. These deviations are clearly reflected in the response of the wide-field Horizontal Cells, thus, providing very specific evidence that the movement detector theory developed here can be applied to motion detection in the fly. The characteristic dynamic features of the theoretically predicted and the experimentally determined cellular responses are exploited to estimate the time constant of the movement detector filter.

\section{Introduction}

The evaluation of motion information is a precondition for the solution of many information processing tasks. There is now good evidence that movement detection in humans is based on essentially the same principle as has originally been proposed for the insect visual system (e.g. van Doorn and Koenderink 1982a, b; van Santen and Sperling 1984; Wilson 1985; Baker and Braddick 1985). Roughly speaking, the mechanism underlying movement detection is nonlinear and local. It is based on the multiplication-like interaction of the appropriately filtered signals of neighbouring retinal input channels. Since this principle might be of widespread importance in the animal kingdom, the visual system of the fly can be regarded as a model system and its methodological advantages 
exploited to gain knowledge about certain aspects of the processing of motion information.

This notion, however, needs some further qualification. It should be made quite explicit that the equivalence of the mechanisms underlying movement detection in insects and vertebrates concerns the principle algorithm only, i.e. the computations by which this information processing task can be formally accomplished. This does not exclude that the actual neuronal representation of this algorithm turns out to be quite different in the insect and the vertebrate brain. Common principles of information processing in different species might be quite obvious at the algorithmic level but obscured in the cellular details of the underlying neuronal wiring scheme. This underlines the appeal of an algorithmic approach to information processing problems such as motion detection.

Originally, the formal expression for the inputoutput relationship of the insect motion detection system made allowance for the time-averaged response only (e.g. Hassenstein and Reichardt 1956; Reichardt 1957, 1961; Reichardt and Varjú 1959; Varjú 1959; Poggio and Reichardt 1973; see also Buchner 1984). Despite its predictive power this approach, thus, suffers from a serious disadvantage, because it can only be applied appropriately to patterns which move at a constant velocity. This holds also for the variants of this motion detection model which have been used in human psychophysics (van Santen and Sperling 1984; Wilson 1985; for a discussion of different models which are, however, mathematically equivalent at the movement detector output: see Adelson and Bergen 1985; van Santen and Sperling 1985).

Only recently a different mathematical approach has been employed by Reichardt and Guo (1986) to derive a formal expression for the movement detector response to non-stationary stimulus conditions. In principle, this approach is characterized by a transition from a detector array with a finite spatial sampling base to a continuous field of detectors to which the techniques of analysis can be applied in both space and time. Although this formalism allows one to calculate instantaneous detector responses to instationary pattern motion, it can only be applied in a limited dynamic range. As will be shown in the present study, the model response to a given pattern reflects the characteristic features of the experimentally determined output of the motion detection system only as long as the pattern velocity and its time derivatives are sufficiently small. Otherwise significant deviations may occur. It is the main objective of the present study to overcome these limitations. For this end the approach taken by Reichardt and Guo (1986) will be generalized here. The resulting time-dependent formal expression for the response of an elementary movement detector can now be applied to one-dimensional patterns moving in an almost arbitrary way. As the only qualification, the time-dependent input function of the movement detector should possess a convergent Taylor series for any value of the spatial coordinate.

The significance of this generalized movement detector theory can be tested experimentally. Depending on the dynamic range of pattern motion, it predicts characteristic deviations of the movement detector output from being proportional to the velocity of the stimulus pattern. These deviations are even clearly reflected in the time course of the spatially integrated response of a retinotopic array of movement detectors. Since the relatively large tangential neurones of the third visual ganglion of the fly receive motion specific retinotopic input from considerable parts of the visual field (Hausen 1981), their methodological advantages will be used to corroborate that the motion detector theory presented here can be applied to motion detection in the fly. Finally, the time constant of the movement detector filter will be estimated by taking advantage of the aforementioned qualitative changes of the response profiles.

\section{Materials and Methods}

\subsection{Electrophysiology}

The electrophysiological measurements were carried out with wild type female blowflies, Calliphora erythrocephala (Meig.). All animals were obtained 2-10 days post eclosion from laboratory cultures of the institute.

The preparation follows the routine for intracellular recording in the fly optic lobes developed previously (see Hausen 1982a). The test fly was immobilized and the rear of its head cuticle was opened in order to gain access to the lobula complex of the right optic lobe. The Horizontal Cells of the right lobula plate were recorded from intracellularly and in most cases subsequently stained with Lucifer Yellow in order to allow unambiguous identification of the cell. The cells were usually penetrated in their axonal regions. The electrodes were pulled with a P-77 BrownFlaming Micropipette Puller (Sutter Instruments). When filled with $1 M$ potassium acetate solution, the electrodes had resistances of $20-60 \mathrm{M} \Omega$. The recorded graded potentials were averaged with a signal averager and subsequently plotted on a X-Y-recorder. The electrophysiological techniques are described in more detail elsewhere (Egelhaaf 1985a, b).

\subsection{Visual Stimulation}

The fly's head was positioned in the centre of a cylindrical pattern, its diameter and height amounting 
to $70 \mathrm{~mm}$ and $50 \mathrm{~mm}$, respectively. This corresponds to a vertical angular extent of the stimulus of about $\pm 35^{\circ}$, when the fly is suspended in the middle of the cylinder. The pattern cylinder was opened behind the fly in order to allow access to the animal's brain with the electrode; its angular horizontal extent amounted to $\pm 120^{\circ}$ with respect to the longitudinal axis of the head. The cylinder was covered with a vertical sinewave grating. It was illuminated from above with a fibre optic ring light connected with a cold light source which operated at $3400 \mathrm{~K}$ colour temperature. The mean luminance and the contrast of the pattern amounted to about $1100 \mathrm{~cd} / \mathrm{m}^{2}$ and 0.32 , respectively. The pattern cylinder was oscillated sinusoidally about its vertical axis with variable oscillation frequencies and amplitudes, as will be specified in the result section.

\subsection{Computer Simulation}

The model simulations shown in Fig. 5 were carried out with a Hewlett-Packard 9826 computer. The programmes were written in BASIC. The spatially integrated movement detector responses shown in Fig. 2 and the contour plot of Fig. 3 were calculated on an IBM-XT using the ASYST-software (Keithley Instruments).

\section{Functional Representation of a Moving Pattern in the Output of an Elementary Movement Detector}

A single detector of the type as has previously been proposed to underly motion detection in insects (Hassenstein and Reichardt 1956; Reichardt 1957, 1961; Reichardt and Varjú 1959; Varjú 1959) is illustrated in Fig. 1 in its most simplified form. It has two input channels which are spatially separated by a small interval $\Delta x$. Hence, a movement detector has a welldefined orientation in space. For simplicity, the input channels of the movement detector are assumed to have point-like receptive fields. Of course, it would be more realistic with respect to the insect eye to convolve the stimulus pattern with the experimentally determined angular sensitivity distribution of the input channels (for review see Hardie 1985). This, however, only alters the amplitudes of the high spatial frequency Fourier components of the pattern (see Götz 1965) but does not affect the principle mechanism of movement detection.

The detector consists of two subunits that are mirror images of each other. These subunits share the same input signals $F(x, t)$ and $F(x+\Delta x, t)$, where $x$ and $t$ denote the spatial variable and time, respectively. It has been assumed, for convenience, that the coordinate system in which $F(x, t)$ is defined is aligned with the

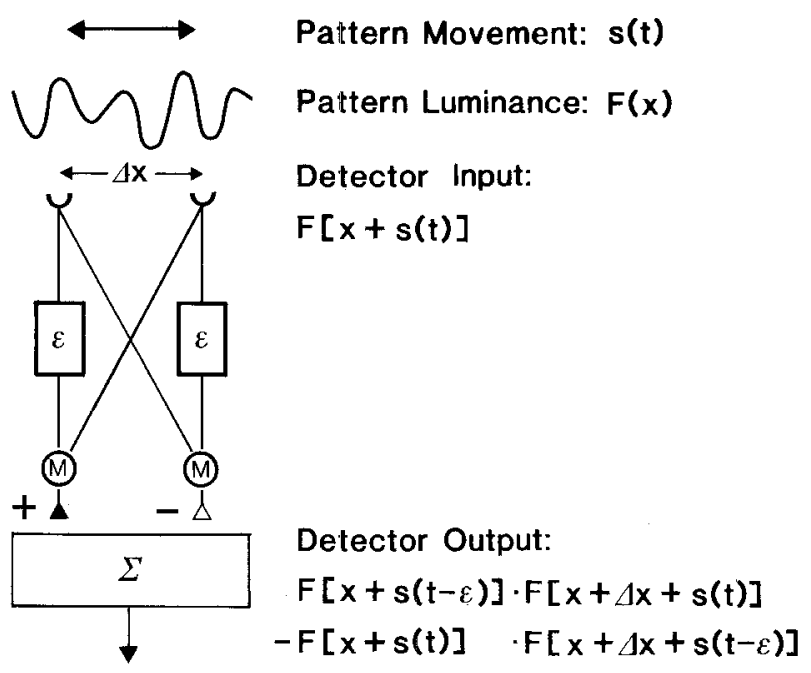

Fig. 1. Schematic representation of an elementary movement detector. It consists of two mirror-inverted subunits. In the simplest version of the detector model the input signal of one branch of each subunit is delayed by a brief time interval $\varepsilon$. In each subunit the delayed signal originating from one retinal location is multiplied with the undelayed signal of the neighbouring input channel. The final detector output is given by the difference of the subunits' output. The formal expressions for the pattern motion and luminance, the receptor input, as well as the detector output are given in their most general form. For further details see text

movement detector axis. The signal of one branch of each subunit passes through a linear temporal filter. For simplicity, this filter will be approximated by a pure delay $\varepsilon$. As will be discussed on the basis of computer simulations in Sect. 6, this does not affect the principle conclusions to be drawn here. In each subunit the delayed signal originating from one retinal location is multiplied with the instantaneous signal of the neighbouring input channel. The final output of the detector is given by the difference between the subunit outputs

$F(x, t-\varepsilon) \cdot F(x+\Delta x, t)-F(x+\Delta x, t-\varepsilon) \cdot F(x, t)$.

If one considers an arbitrary one-dimensional pattern moving along the detector axis with an instantaneous velocity $d s(t) / d t$, where $s(t)$ represents the time-dependent spatial displacement of the pattern, the movement detector input function has the following form

$F(x, t)=F[x+s(t)]$.

Hence the signals at the two input stages of a movement detector are given by $F[x+s(t)]$ and $F[x+\Delta x+s(t)]$, respectively (see Fig. 1). If $\Delta x$ is small, $F[x+\Delta x+s(t)]$ may be approximately derived from $F[x+s(t)]$ by adding the first term of a Taylor series 
developed about $x$. One obtains

$F[x+\Delta x+s(t)] \approx F[x+s(t)]+\frac{\partial}{\partial x} F[x+s(t)] \cdot \Delta x$.

For most practical purposes, (3) represents a satisfactory approximation, since under normal conditions the spatial sensitivity distribution of the photoreceptors prevents the higher order terms of the Taylor series from becoming too large. Taking (2) and (3) into account and letting $\Delta x \rightarrow 0(1)$ can be rewritten

$$
\begin{aligned}
d D(x, t)= & \left\{F[x+s(t-\varepsilon)] \cdot \frac{\partial}{\partial x} F[x+s(t)]\right. \\
& \left.-F[x+s(t)] \cdot \frac{\partial}{\partial x} F[x+s(t-\varepsilon)]\right\} d x
\end{aligned}
$$

with $d D(x, t)$ the time dependent output at $x$.

$F[x+s(t-\varepsilon)]$ may be obtained by a Taylor series developed about $t$. In general, it cannot be taken for granted that the higher order terms of this series are sufficiently small. In contrast to (3) they, therefore, are taken into account and the Taylor series is assumed to converge to $F[x+s(t-\varepsilon)]$. It may then be represented by the following expression

$F[x+s(t-\varepsilon)]=\sum_{n=0}^{\infty} \frac{(-\varepsilon)^{n}}{n !} \cdot \frac{\partial^{n}}{\partial t^{n}} F[x+s(t)]$.

Since $F[x+s(t)]$ is a composite function of the variable $t$, (5) can be rewritten (see e.g. Gradshteyn and Ryzhik 1965)

$$
\begin{aligned}
F[x+s(t-\varepsilon)]= & \sum_{n=0}^{\infty} \frac{(-\varepsilon)^{n}}{n !} \sum_{n=\Sigma v k_{v} ; m=\Sigma k_{v}} \frac{n !}{\prod_{v} k_{v} !} \\
& \times \frac{\partial^{m}}{\partial s^{m}} F[x+s(t)] \cdot \prod_{v}\left(\frac{1}{v !} \cdot \frac{d^{v} s}{d t^{v}}\right)^{k_{v}} .
\end{aligned}
$$

The second summation symbol indicates summation over all solutions of the equation $n=\sum v k_{v}$ where $v$ and the $k_{v}$ are integers satisfying the conditions $1 \leqq v<\infty$ and $0 \leqq k_{v}<\infty$, respectively. The product signs in (6) mean the multiplication over all $v$ for which the condition $k_{v} \neq 0$ is satisfied. $m$ is then determined by the equation $m=\sum k_{v}$. Since with $n \rightarrow \infty$ also $m \rightarrow \infty$, the double sum in (6) implies summation over all integers $n$ and $m$ which satisfy the inequalities $0 \leqq n<\infty$ and $0 \leqq m<\infty$, respectively. The summands constituting this expression, therefore, can be rearranged and (6) can be rewritten.

$$
\begin{aligned}
F[x+s(t-\varepsilon)]= & \sum_{m=0}^{\infty} \frac{\partial^{m}}{\partial s^{m}} F[x+s(t)] \cdot \frac{1}{m !} \sum_{m=\Sigma k_{v}} \frac{m !}{\prod_{v} k_{v} !} \\
& \times \prod_{v}\left(\frac{(-\varepsilon)^{v}}{v !} \cdot \frac{d^{v} s}{d t^{\nu}}\right)^{k_{v}} .
\end{aligned}
$$

In this expression the second summation sign indicates summation over all solutions in non-negative integers of the equation $m=\sum k_{v}$ with the index variable $v$ satisfying the condition $1 \leqq v<\infty$. Again, the product signs in (7) stand for the multiplication over all $v$ for which the condition $k_{v} \neq 0$ is satisfied. By applying the polynomial theorem this expression can be greatly simplified

$$
\begin{aligned}
F[x+s(t-\varepsilon)]= & \sum_{m=0}^{\infty} \frac{\partial^{m}}{\partial s^{m}} F[x+s(t)] \\
& \times \frac{1}{m !}\left[\sum_{v=1}^{\infty} \frac{(-\varepsilon)^{v}}{v !} \cdot \frac{d^{v} s}{d t^{\nu}}\right]^{m} .
\end{aligned}
$$

For $\frac{\partial}{\partial x} F[x+s(t-\varepsilon)]$ one obtains correspondingly

$$
\begin{aligned}
\frac{\partial}{\partial x} F[x+s(t-\varepsilon)]= & \sum_{m=0}^{\infty} \frac{\partial}{\partial x}\left\{\frac{\partial^{m}}{\partial s^{m}} F[x+s(t)]\right\} \\
& \times \frac{1}{m !}\left[\sum_{v=1}^{\infty} \frac{(-\varepsilon)^{v}}{v !} \cdot \frac{d^{v} s}{d t^{v}}\right]^{m} .
\end{aligned}
$$

If one further takes into account that

$\frac{\partial}{\partial s} F[x+s(t)]=\frac{\partial}{\partial x} F[x+s(t)]$,

(4) can be reformulated resulting in the following expression for the time-dependent movement detector response

$$
\begin{aligned}
d D(x, t)= & \sum_{m=1}^{\infty}\left[\frac{\partial F}{\partial x} \cdot \frac{\partial^{m} F}{\partial x^{m}}-F \frac{\partial^{m+1} F}{\partial x^{m+1}}\right] \\
& \times \frac{1}{m !}\left[\sum_{\nu=1}^{\infty} \frac{(-\varepsilon)^{\nu}}{v !} \cdot \frac{d^{v} s}{d t}\right]^{m} d x .
\end{aligned}
$$

Taking into account the Taylor series of $s(t-\varepsilon)$

$s(t-\varepsilon)=\sum_{v=0}^{\infty} \frac{(-\varepsilon)^{v}}{v !} \cdot \frac{d^{v}}{d t^{v}} s(t)$

one finally obtains from (11) for the movement detector output

$$
\begin{aligned}
d D(x, t)= & \sum_{m=0}^{\infty}\left[\frac{\partial F}{\partial x} \cdot \frac{\partial^{m} F}{\partial x^{m}}-F \frac{\partial^{m+1} F}{\partial x^{m+1}}\right] \\
& \times \frac{1}{m !}[s(t-\varepsilon)-s(t)]^{m} d x .
\end{aligned}
$$

One important conclusion can be drawn from (11) and (13): A movement detector of the type discussed here is not a pure velocity sensor, since its output is not determined exclusively by the pattern velocity. Instead, its response can be represented by a power series of the time-dependent displacement of the pattern during the delay time of the movement detector filter. According to (12) this displacement corresponds to the weighted sum of the pattern velocity 
and all its higher order time derivatives. This patternindependent component of each term of the series is weighted by a factor which depends in a non-linear way on the pattern texture and its spatial derivatives. In addition, this factor depends on time. How many terms of the series of (11) and (13) are required to approximate the movement detector output sufficiently well depends, of course, on the specific properties of the pattern under consideration and its particular movement. If only the first term of this series is taken into account and if the Taylor expansion of $s(t-\varepsilon)$ is terminated after only the first derivative term one obtains

$d D(x, t)=-\varepsilon \frac{d s}{d t}\left[\left(\frac{\partial F}{\partial x}\right)^{2}-F \frac{\partial^{2} F}{\partial x^{2}}\right] d x$.

This expression is identical with the "first approximation" of the response of an elementary movement detector as has been derived by Reichardt and Guo (1986). It will be shown experimentally in this paper that at least for the fly movement detection system this first approximation may not suffice under certain stimulus conditions to explain the movement detector response and that the higher order terms of (11) and (13) need to be taken into account.

It should be noted that the movement detector theory developed here is concerned with one spatial dimension only. It has been extended, so far, to two dimensions only in the case where the first approximation [see (14)] of the movement detector response can be applied. Under these conditions the velocity vector of the stimulus can be related by a two-dimensional symmetric tensor to the vector formed by the output of a pair of differently oriented movement detectors. The elements of the tensor are functions of the stimulus pattern (Reichardt 1985). This rather simple relation fails, of course, under stimulus conditions where the generalized movement detector theory [(11) and (13)] has to be applied.

\section{Theoretical Predictions: \\ The Response of a Spatially Integrated Array of Movement Detectors to Sinusoidal Pattern Motion}

In order to transfer the theoretical formulation of the movement detector output as derived in the previous section into predictions which eventually can be tested experimentally two specifications will be made. They were chosen to reveal in a simple way the significance of the higher-order terms beyond the first approximation of the detector theory [see (11) and (13)].

i) The output of a one-dimensional retinotopic array of movement detectors rather than of a single detector will be taken into account. This simplifies the situation greatly, since integrating the movement de- tector output between appropriately chosen limits with respect to the spatial coordinate eliminates the time dependence of the pattern-dependent component of each term of the series of (13). As a consequence, each term of this series is separable into a pattern dependent constant and a time-dependent function which does not depend on the texture of the pattern (see also Reichardt and Guo 1986). Apart from this theoretical advantage, the consideration of the spatially integrated movement detector response provides also methodological advantages. Wide-field tangential neurones of the third visual ganglion of the fly receive input from large arrays of horizontally oriented elementary movement detectors (see Sect. 5). Since these cells are relatively large, they can be recorded from most easily of all higher order visual interneurones of the fly's brain.

ii) A moving one-dimensional sine-wave pattern was chosen as a pattern function $F(x, t)$ in the theoretical predictions and, accordingly, in the electrophysiological experiments. This specific time-dependent pattern function then reads

$F(x, t)=I+\Delta I \sin \frac{2 \pi}{\lambda}[x+s(t)]$,

where $I$ denotes the mean intensity, $\Delta I$ the modulation, and $\lambda$ the spatial wavelength of the pattern.

With a moving sine-wave grating as input function one obtains from (13) for the movement detector output

$$
\begin{aligned}
d D(x, t)= & \frac{2 \pi}{\lambda}\left\{\left\{\Delta I^{2}+I \cdot \Delta I \sin \frac{2 \pi}{\lambda}[x+s(t)]\right\}\right. \\
& \times \sum_{m=0}^{\infty}\left(\frac{2 \pi}{\lambda}\right)^{2 m+1} \cdot[s(t-\varepsilon)-s(t)]^{2 m+1} \\
& \times \frac{(-1)^{m}}{(2 m+1) !} \\
& +I \cdot \Delta I \cos \frac{2 \pi}{\lambda}[x+s(t)] \cdot \sum_{m=1}^{\infty}\left(\frac{2 \pi}{\lambda}\right)^{2 m} \\
& \left.\times[s(t-\varepsilon)-s(t)]^{2 m} \cdot \frac{(-1)^{m+1}}{(2 m) !}\right\} d x .
\end{aligned}
$$

Integrating (16) over an integer multiple $n$ of the spatial period $\lambda$ yields

$$
\begin{aligned}
D(t)= & \frac{2 \pi}{\lambda} \Delta I^{2} \cdot \sum_{m=0}^{\infty}\left(\frac{2 \pi}{\lambda}\right)^{2 m+1} \\
& \times[s(t-\varepsilon)-s(t)]^{2 m+1} \cdot \frac{(-1)^{m}}{(2 m+1) !} \cdot C,
\end{aligned}
$$

where $C=n \lambda$. Taking into account the series expansion for the sine function one finally obtains for the response of a spatially integrated array of movement 
Output of a Detector Array:

$$
\mathrm{R}(\mathrm{t})=\frac{2 \pi}{\lambda} \Delta \mathrm{I}^{2} \cdot \sin \frac{2 \pi}{\lambda}[\mathrm{s}(\mathrm{t}-\varepsilon)-\mathrm{s}(\mathrm{t})] \cdot \mathrm{n} \lambda
$$

$$
\frac{\varepsilon}{T}=\frac{1}{30}
$$
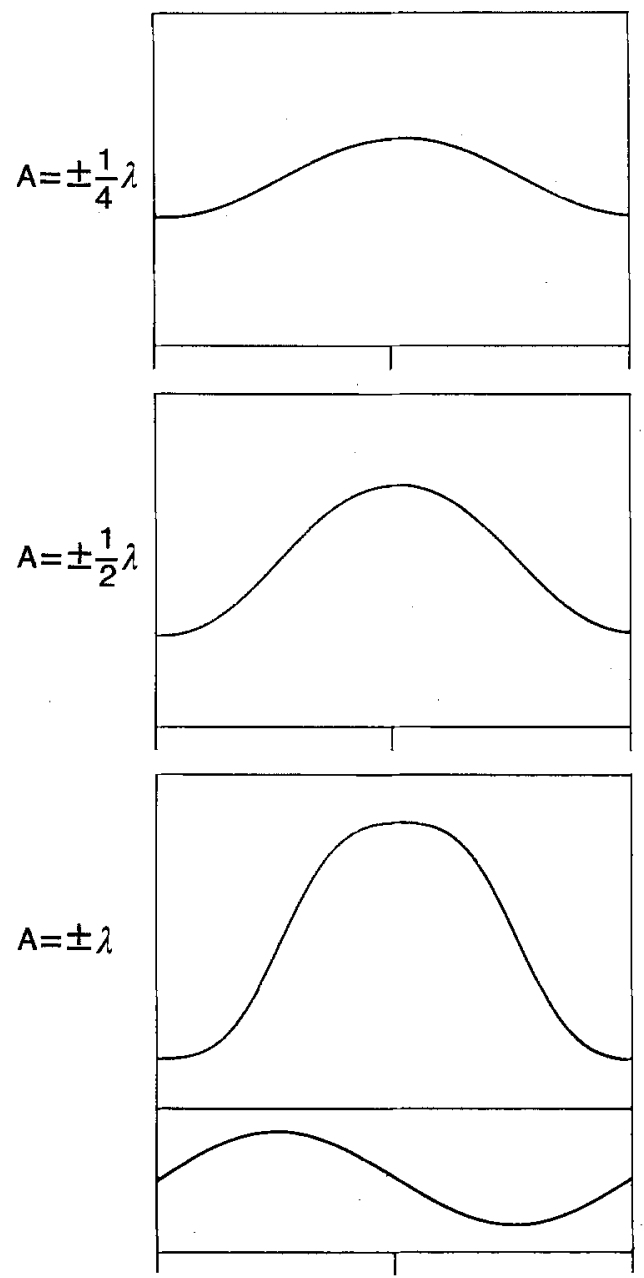

\section{Pattern Movement:}

$$
s(t)=A \cdot \sin \frac{2 \pi}{T} t
$$
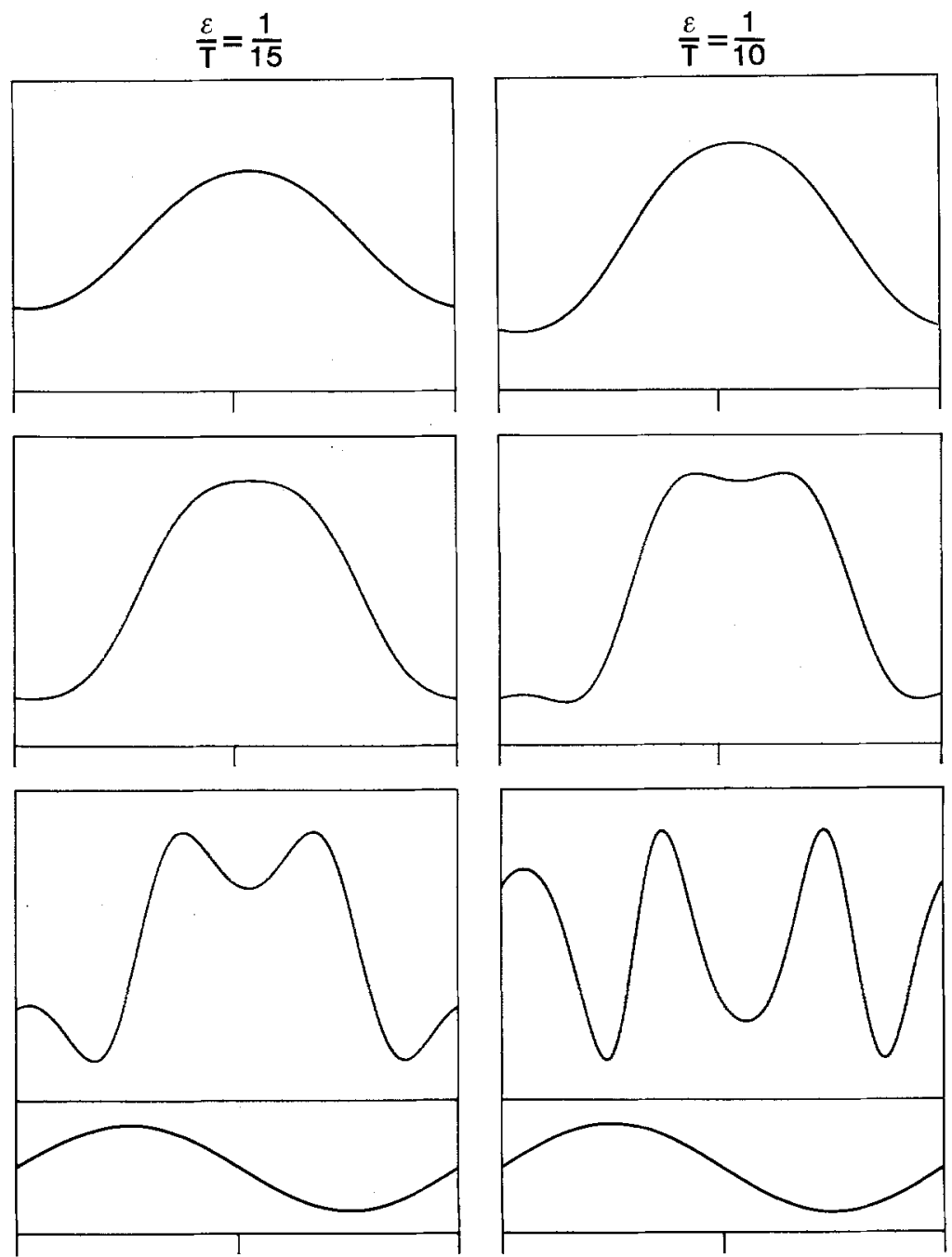

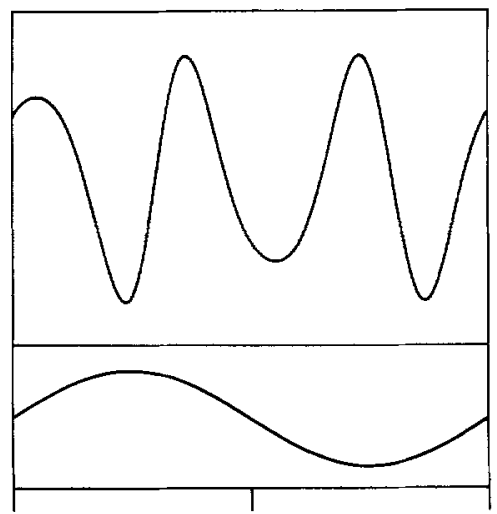

Time

Fig. 2. Response of a spatially integrated array of movement detectors. The curves are based on equation (19) which is given at the upper margin of the figure. The stimulus pattern consists of a one-dimensional sine-wave grating which is oscillated sinusoidally. In the different curves the oscillation amplitude $A$, given in units of the spatial wavelength $\lambda$ of the pattern, and the ratio of the movement detector delay $\varepsilon$ and the temporal period $T$ are varied. The bottom traces indicate the time-dependent deviation of the pattern from its mean position. The response is about proportional to pattern velocity only for small $\varepsilon / T$ and $A / \lambda$. For larger $\varepsilon / T$ and $A / \lambda$ characteristic deformations of the response become visible. These are the more pronounced the larger $\varepsilon / T$ and $A / \lambda$. Further abbreviations: $\Delta I$; degree of modulation; $n$ : number of cycles over which the movement detector response is integrated

detectors

$D(t)=\frac{2 \pi}{\lambda} \Delta I^{2}\left\{\sin \frac{2 \pi}{\lambda}[s(t-\varepsilon)-s(t)]\right\} \cdot C$.

For this particular pattern function the movement detector response can also be calculated directly without employing approximation techniques. This leads, of course, to exactly the same expression, if one takes into account that the distance $\Delta x$ between the inputs of the movement detector is assumed in the present approximation to be infinitesimally small. Otherwise the term $\frac{2 \pi}{\lambda}$ in (18) has to be replaced by $\sin \frac{2 \pi}{\lambda} \Delta x$ (for the significance of this "geometrical interference" term, see Varjú 1959; Götz 1964). In any case, this term is a constant for a given pattern which does not affect the time course of the response. 
From (18) it can be seen that also in the special case of a moving sine-wave grating the movement detector response is not proportional to pattern velocity even after spatial integration. Rather it is proportional to the sine of the time-dependent displacement of the pattern during the delay time of the movement detector filter which corresponds to the weighted sum of the pattern velocity and all its higher order time derivatives [see (12)]. If a special time-dependent displacement function is chosen and the sine-wave grating oscillated sinusoidally with an amplitude $A$ and a temporal period $T$ one obtains from (18)

$D(t)=\frac{2 \pi}{\lambda} \Delta I^{2} \sin \left\{\frac{2 \pi}{\lambda} A\left[\sin \frac{2 \pi}{T}(t-\varepsilon)-\sin \frac{2 \pi}{T} t\right]\right\} \cdot C$.

By application of basic trigonometric rules (19) can be rewritten

$$
\begin{aligned}
D(t)= & -\frac{2 \pi}{\lambda} \Delta I^{2} \\
& \times \sin \left\{\frac{2 \pi}{\lambda} A\left[2 \cos \frac{2 \pi}{T}\left(t-\frac{\varepsilon}{2}\right) \cdot \sin \pi \frac{\varepsilon}{T}\right]\right\} \cdot C
\end{aligned}
$$

which allows to draw a conclusion that eventually can be tested experimentally: The spatially integrated time-dependent movement detector response to sinusoidal oscillation of a sine-wave pattern is not, in general, a cosine function but the sine of a cosine function. Only if the ratio of oscillation amplitude $A$ and spatial wavelength $\lambda$ and/or the ratio of filter delay $\varepsilon$ and temporal period $T$ are sufficiently small the sine in (20) may be dropped resulting in a response with a sinusoidal time course. Otherwise considerable deformations in the response profiles occur, which are the more pronounced the larger $A / \lambda$ and $\varepsilon / T$.

In Fig. 2 these qualitative changes of the time course of the spatially integrated movement detector response are shown. They were obtained from (19) for three oscillation amplitudes and frequencies, respectively. In accordance with the above conclusions, the response is a simple cyclic function being approximately proportional to pattern velocity only for small oscillation amplitudes and frequencies. With increasing amplitudes and/or frequencies the response becomes gradually deformed. At first the response peaks flatten. Eventually characteristic indentations become visible in the time course of the response which are the more pronounced the higher the oscillation frequency and amplitude. It should be noted that these qualitative changes occur while the response attains its maximum amplitude.

These deformations of the response profiles at high oscillation frequencies and amplitudes can be quanti- fied by calculating the "distortion factor." This measure represents the relative contribution of the higher order harmonics to the response of the system and is defined as follows

$D F=\sqrt{\frac{\sum_{v=2}^{\infty}\left|G_{v}\right|^{2}}{\sum_{v=1}^{\infty}\left|G_{v}\right|^{2}}}$.

In this expression, the $G_{v}$ form the amplitude spectrum of the spatially integrated movement detector response $D(t)$. The distortion factor ranges between 0 and 1 . It is displayed in the contour plot of Fig. 3 as a function of the oscillation frequency and amplitude, respectively.

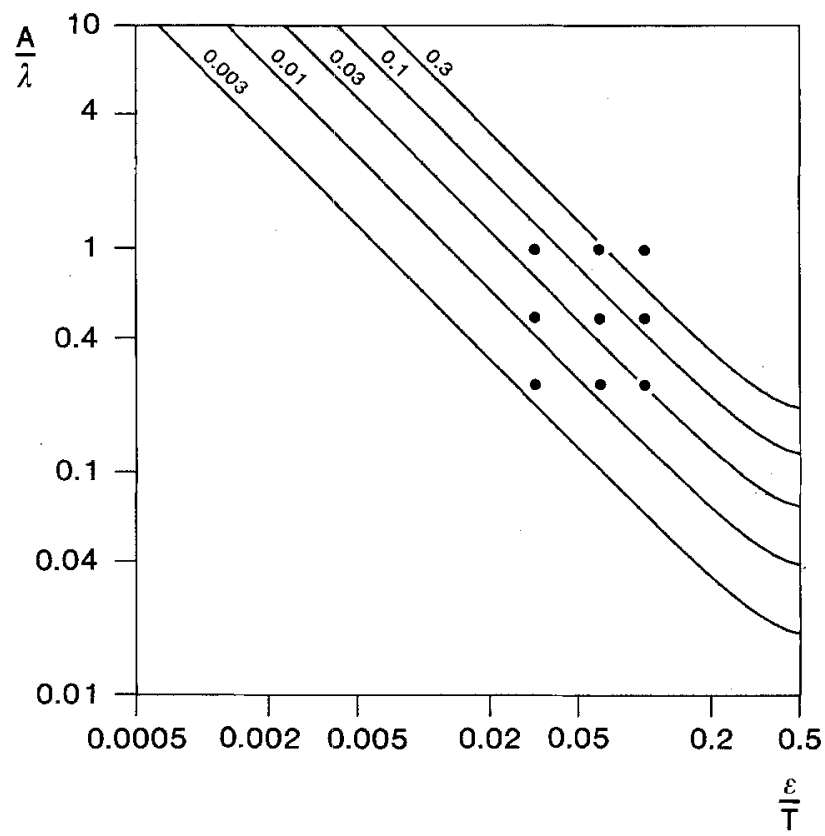

Fig. 3. Contour plot of the distortion factor [see (21)] derived from the spatially integrated movement detector response to sinusoidal oscillation of a sine-wave grating [see (19)]. The distortion factor is calculated as a function of the ratio of the movement detector delay $\varepsilon$ and the temporal period $T$ as well as the oscillation amplitude $A$ and the spatial wavelength $\lambda$ of the pattern. Both $\varepsilon / T$ and $A / \lambda$ were varied over a range of three decades. The lines represent iso-distortion-factor lines and subdivide the $\varepsilon / T-A / \lambda$-plane in domains representing different ranges of distortion factors. The corresponding distortion factors are indicated in the figure. The points correspond to the response profiles shown in Fig. 2. They were drawn in the contour plot to facilitate an assignment of these response profiles and their corresponding distortion factors. On this basis it can be concluded that in the dynamic range corresponding to distortion factors smaller than 0.03 the spatially integrated movement detector response can be accounted for sufficiently well by the first approximation of the movement detector theory [see (14)]. For larger distortion factors the higher order terms of the series representing the movement detector output [see (13)] need to be taken into account 
As in Fig. 2, the oscillation frequency is given in units of the movement detector time constant and the amplitudes in units of the spatial wavelength of the pattern. Both parameters were varied over three decades. To facilitate establishing a relation between the time course of the spatially integrated movement detector responses under the different stimulus conditions (see Fig. 2) and their respective distortion factors the corresponding points in the contour plot are marked. On this basis it can be concluded that for distortion factors below 0.03 the spatially integrated movement detector response to sinusoidal pattern motion can be regarded as about proportional to pattern velocity. The dynamic range of stimulus motion in which the first approximation of the movement detector theory [see (14) and Reichardt and Guo 1986] can be applied can thus be inferred from the contour plot. In the dynamic range where the response profiles flatten the corresponding distortion factors range between 0.03 and 0.1 (compare Figs. 2 and 3). For even larger distortion factors the characteristic indentations in the response profiles become visible. In the corresponding dynamic range of stimulus motion the first approximation of the movement detector theory is no longer sufficient and the higher order terms of the series of (13) have to be taken into account.

\section{Experimental Test: \\ Time Course of the Horizontal Cell Response}

If movement perception in the fly were based on the motion detection scheme as outlined in Sect. 3 , the theoretically predicted qualitative changes of the spatially integrated response of this type of movement detector should be reflected somewhere in the output of the fly's motion information processing system. This kind of distortions have been observed in the time course of the optomotor turning reaction to oscillating periodic large-field gratings at high oscillation frequencies (Guo and Reichardt 1987). Therefore, we were encouraged to engage in a more systematic analysis of this phenomenon. The lobula plate widefield neurones were employed for this analysis instead of the behavioural level, since by just these neurones the spatial integration of the local movement information is accomplished.

In the lobula plate, the posterior part of the third visual ganglion of the fly (see inset of Fig. 4), there reside several directionally selective motion sensitive large-field tangential neurones. These receive input by a large number of retinotopically organized columnar elements from the entire visual field of an eye or at least from a considerable part of it (Hausen 1982a, b).
Although the latter could not be characterized electrophysiologically so far, they are believed to represent local movement detectors (for a discussion of the evidence for this, see Hausen 1981; Egelhaaf 1985c). The lobula plate tangential neurones, therefore, seem to be a good system for studying the response of a spatially integrated array of movement detectors. It should be emphasized, however, that all of these neurones, known so far, do not summate their input linearly as was assumed in the theoretical predictions of Sect. 4. Instead they reveal characteristic nonlinear spatial integration properties (Hausen 1982b; Hengstenberg 1982; Egelhaaf 1985a, b). Nevertheless, it can be shown (see Sect. 6) that these nonlinearities affect the response only little as far as its time-course is concerned.

Among the lobula plate tangential cells the three so-called Horizontal Cells are the main output neurones of the optic lobes which control the optomotor large-field turning reaction (Hausen 1981; Reichardt et al. 1983; Egelhaaf 1985a, c; Wehrhahn 1985). They have been analysed in this study with respect to their dynamic response properties, since they can be recorded from intracellularly relatively easily. Similar dynamic response properties were found in other lobula plate large-field tangential cells.

The activity of the Horizontal Cells was recorded intracellularly while the fly was stimulated with a vertical sine-wave grating. The grating was oscillated sinusoidally in the horizontal direction with different frequencies and amplitudes. A representative selection of records from these experiments is shown in Fig. 4. They were obtained from one of the three Horizontal Cells, i.e. the right South Horizontal Cell (see inset of Fig. 4) of a single test fly by averaging the cell's de- and hyperpolarizations in response to several stimulation cycles. It should be noted that graded membrane potential changes rather than regular spike trains are the prominent response mode of the Horizontal Cells to ipsilateral motion (Hausen 1982a, b). As is known for long, the Horizontal Cells are depolarized by movement with a constant velocity from front-to-back and are hyperpolarized by motion in the opposite direction (Hausen 1982a, b). This may even be true, when the velocity of the pattern changes continuously as is the case during sinusoidal oscillation. This is illustrated by two of the sample records of Fig. $4(2 \mathrm{~Hz}$, $A=10^{\circ}$ and $8 \mathrm{~Hz}, A=2.5^{\circ}$ ): The cell steadily depolarizes during front-to-back motion until the membrane potential reaches a more or less pronounced plateau level. It hyperpolarizes again as soon as the pattern reverses its direction of motion and finally attains a membrane potential below the cell's resting level. Similar records have already been described before (Reichardt et al. 1983; Egelhaaf 1985a). 

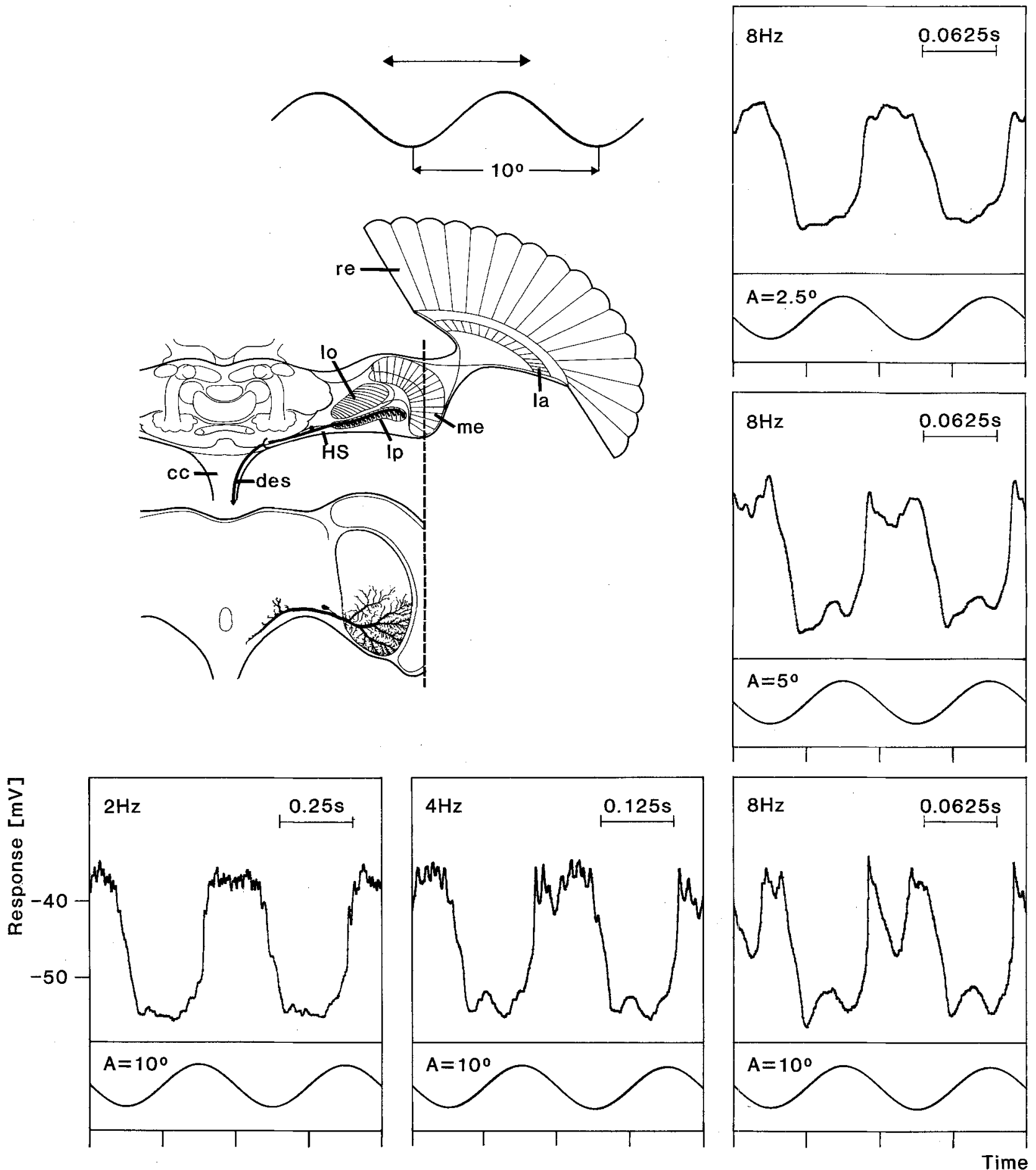

Fig. 4. Responses of a South Horizontal Cell to sinusoidal oscillation of a sine-wave grating with various oscillation frequencies and amplitudes. The position of the cell in the frontal layers of the lobula plate $(l p)$, the posterior part of the third visual ganglion is indicated in the schematic horizontal cross-section through the eyes, optic lobes, and brain of the fly (modified from Hausen 1981). The anatomy of a South Horizontal Cell is shown in frontal projection in the lower part of the inset (by courtesy K. Hausen). The angular horizontal extent of the sine-wave pattern amounted to $\pm 120^{\circ}$. Its spatial wavelength was $10^{\circ}$. As is indicated in the sub-figures the oscillation frequency and amplitude $(A)$ were varied in the different experiments. Note the different time scales! At the bottom of each record the time-dependent deviation of the stimulus pattern from its mean position is shown. Downward and upward deflections indicate clockwise and counterclockwise motion, respectively. The elctrophysiological data represent response averages of the membrane potential changes of a single South Horizontal Cell. They were obtained from 16 repetitions of the respective stimulus sequences. The electrophysiological records illustrate that the Horizontal Cell is depolarized by front-to-back motion and hyperpolarized by motion in the opposite direction only for small oscillation frequencies and/or amplitudes. Beyond this dynamic range the membrane potential reveals hyperpolarizing deflections even during clockwise motion and depolarizing deflections during counterclockwise motion.

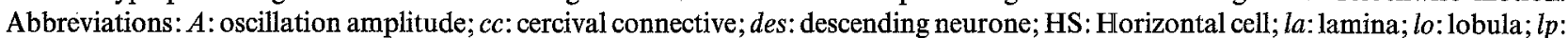
lobula plate; $m e$ : medulla; re: retina 
In accordance with the theoretical predictions of the previous sections this simple cyclic response pattern of the Horizontal Cells to sinusoidal pattern oscillation is only generated, if (for a given spatial wavelength and oscillation amplitude) the oscillation frequency (e.g. $2 \mathrm{~Hz}$ in Fig. 4) or (for a given oscillation frequency) the ratio of oscillation amplitude and spatial wavelength of the pattern (e.g. $\lambda / 4$ in Fig. 4) are sufficiently small. Otherwise the time-course of the membrane potential changes may become more complicated. If the oscillation frequency or amplitude is increased the stimulus-evoked membrane potential profiles become gradually deformed (see Fig. 4). Similar indentations as were predicted in Sect. 4 (see Fig. 2) become visible about halfway between the steeply rising and falling flanks of the membrane potential. Note that these characteristic indentations emerge during both the depolarizing as well as the hyperpolarizing phase of the membrane potential cycle. They are the more pronounced the higher the oscillation frequency or amplitude. In the most extreme example shown in Fig. $4\left(8 \mathrm{~Hz}, A=10^{\circ}\right)$ both the positive and negative indentations almost reach the resting level of the membrane potential. Despite these deformations of the response profiles the amplitude of the response is not much affected within the dynamic range tested here by changes in both oscillation frequency and amplitude. This is just what has been predicted theoretically in the previous section. Higher oscillation frequencies and amplitudes could not be realized with the mechanical stimulation device employed in this study.

On the basis of these records it can thus be concluded that the generally accepted view that the Horizontal Cells are depolarized by front-to-back motion and hyperpolarized by motion in the reverse direction is only true under certain stimulus conditions. For a given pattern the visually induced membrane potential change is about proportional to pattern velocity only within a certain dynamic range of pattern motion. Only in this range the response can be accounted for by the first term of the power series representing the spatially integrated movement detector response to a sinusoidally oscillating sine-wave pattern [see (17)]. Outside this dynamic range the instantaneous membrane potential may show hyperpolarizing deflections even during front-to-back motion; depolarizing deflections may be induced by motion in the opposite direction. This can only be explained on the basis of the movement detector model analysed here, if the higher order terms of the series of (17) are taken into account.

On the whole, these observations are fully in accordance with the theoretical predictions of the previous sections. They, thus, corroborate in a very specific way the motion detection scheme initially proposed on the basis of time averaged data to underly movement detection in insects (Hassenstein and Reichardt 1956; Reichardt 1957, 1961). This is because the model is sufficient also to explain the characteristic dynamic features of motion information as it is represented at the level of the large-field tangential neurones in the third visual ganglion as well as at the behavioural level in the optomotor turning reaction (Guo and Reichardt 1987). Hence, the theoretically derived finding that the spatially integrated output of an array of movement detectors is proportional to pattern velocity only within a certain dynamic range of motion is not an idiosyncrasy of the movement detector model under extreme stimulus conditions. It is rather a prominent feature of the lobula plate tangential neurones under stimulus conditions which can induce almost maximum response amplitudes attainable in these cells (see Fig. 4).

\section{Estimation of the Movement Detector Time Constant}

The dynamic range of stimulus motion in which a movement detector is operational essentially depends on the time constants of its filters. In particular, the relationship of the filter time constant and the pattern velocity as well as its time derivatives determines how many terms of the series of (13) are required to represent the movement detector response faithfully. This implies that the response of a movement detector can only be predicted, if its filter time constant is known.

This characteristic quantity can be estimated by relating the experimentally determined Horizontal Cell response to the corresponding best model fit. Because the time course of the response reveals characteristic qualitative differences under the different dynamic stimulus conditions this correspondence can be established relatively easily. For convenience, this determination of the movement detector time constant will be based on the Horizontal Cell response to sinusoidal oscillation of a sine-wave grating as is shown in Fig. 4. The corresponding model simulations of the Horizontal Cell response were not based on the simple model discussed in Sect. 3 where the model response is obtained by linearly summating the output of a retinotopic array of movement detectors, since this model is inadequate to account for the particular nonlinear spatial integration properties of the Horizontal Cells (Hausen 1982b; Reichardt et al. 1983). Although it can be shown that linear summation of the movement detector response is a good approximation as far as the dynamic response properties of the Horizontal Cells are concerned, the time constant will be derived 
from computer simulations based on a more complicated model. Here the movement detectors feed into a model network which has previously been shown to be sufficient to explain the characteristic spatial integration properties of the Horizontal Cells (see Reichardt et al. 1983; Egelhaaf 1985a).

In brief, this model can be summarized as follows (see inset of Fig. 5): To account for its directional selectivity the model Horizontal Cell (hatched model cell, HS) receives excitatory input from the left subunit of each movement detector and inhibitory input from the right subunit. These synapses are assumed to have a non-linear transmission characteristic. To account for the smaller response amplitude of the Horizontal Cells to hyperpolarizing input as compared with a depolarizing one, the negative and positive response components of the total output of both movement detector subunits are differentially weighted $(0.3: 1)$. Prior to spatial summation the individual movement detector channels are assumed to be shunted via presynaptic inhibition mediated by a hypothetical large-field "pool cell." The latter is proposed to get excitatory input from both movement detector subunits and, consequently, is excited by movement in both horizontal directions. The details of this model which are relevant for an understanding of the computer simulations will be given in the legend of Fig. 5 .

In order to obtain a fairly realistic estimation of the movement detector time constant the model calculated analytically in Sects. 3 and 4 was altered in another way. Instead of a pure delay a low pass filter of second order was used in the computer simulations of the Horizontal Cell response which are shown in Fig. 5. The impulse response function of this filter is shown in the inset of Fig. 5. With this type of filter the details, although not the principle features of the experimentally determined response profiles, are better fitted than with a pure delay. It should be noted, however, that it was not intended to fit the experimental data as closely as possible. This certainly would have been possible by assuming filters of higher order or combinations of different filters in both branches of each movement detector subunit. Instead, it was tried to account for the most prominent features of the time course of the Horizontal Cell response with as few assumptions as possible.

Computer simulations of the Horizontal Cell response based on the model as explained above are displayed in Fig. 5. As in the electrophysiological experiments (see Fig. 4) two different stimulus parameters were varied, i.e. oscillation amplitude and oscillation frequency. To characterize the stimulus in units which eventually can be interpreted with respect to the experimental results, the oscillation amplitude $A$ is given in fractions of the pattern's spatial wavelength $\lambda$ and the temporal period $T$ is related to the filter time constant $\tau$ of the movement detector. Whereas the oscillation amplitude was chosen as in the experiments, the ratio of the filter time constant and the temporal period $\tau / T$ was adjusted as to match the corresponding experimental results as closely as possible. As becomes obvious by comparing Figs. 4 and 5 this can be achieved sufficiently well. Depending on the oscillation frequency one either obtains smooth response profiles or the characteristic indentations which were already analysed theoretically and experimentally in the preceding sections. Again, the larger the oscillation amplitude and/or the ratio of time constant and temporal period the more pronounced are the deformations in the model cell response. Ideally $\tau / T$ should be the same in the best model fits of those experimental results which were obtained under stimulus conditions where the oscillation frequency was held constant while the oscillation amplitude varied. On the other hand, a doubling in oscillation frequency in the experiments should be paralleled by a doubling of $\tau / T$ in the corresponding model simulations. As is indicated in Fig. 5 by the numerical values for $\tau / T$ both predictions are not perfectly satisfied. The deviations from these expectations, however, are sufficiently small, and, therefore, only slightly affect the filter time constants which will be estimated on this basis.

The movement detector time constant can be determined by multiplying $\tau / T$ of the best model fit with the period length used in the corresponding electrophysiological experiment (see Fig. 4). Calculated in this way, the movement detector time constant comes to lie within a range between $5.5 \mathrm{~ms}$ and $8 \mathrm{~ms}$. It should be noted that this difference in the estimated time constant cannot be attributed on the basis of the results shown in Fig. 4 to a dependence of the movement detector time constant on either oscillation frequency or amplitude.

Before one can accept this estimation of the movement detector time constant, one has to ask, whether it depends sensibly on the particular filter which was used in the computer simulations. This was tested by simulating the model with different movement detector filters and determining the time constant in the same way as explained above. In case of a low pass filter of first order the calculated time constant lies in the same range as was obtained with a low pass filter of second order. In the extreme and probably unrealistic case of a pure delay approximating the movement detector filter one obtains, for obvious reasons, values which are larger than the ones obtained with the other filters. Although these values are likely to represent an overestimation of the movement detector time constant, they do not exceed $25 \mathrm{~ms}$ for the experimental data shown in Fig. 4. 

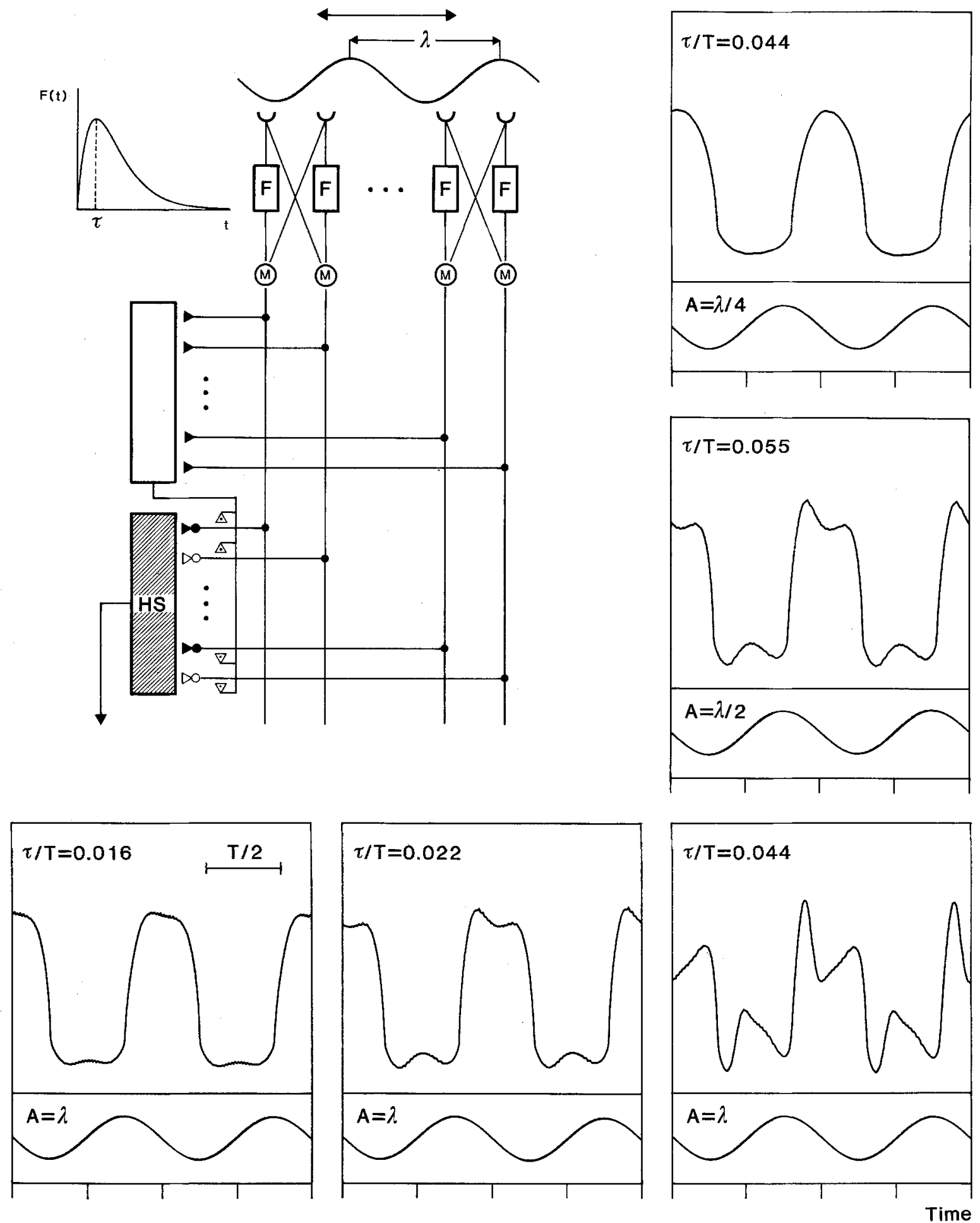

Fig. 5. Responses of a model Horizontal Cell to sinusoidal oscillation of a sine-wave grating with various oscillation frequencies and amplitudes. The computer simulations are based on a model which has previously been proposed in the context of figure-ground discrimination (see inset; Reichardt et al. 1983). To account for its directional selectivity the model Horizontal Cell (HS) receives excitatory input ( $-\infty)$ from the left subunit of each movement detector and inhibitory input ( $-\infty)$ from the right subunit. These synapses are assumed to have a non-linear transmission characteristic. To account for the smaller response amplitudes of the Horizontal Cells to hyperpolarizing input than to depolarizing input, the negative and positive response components of the total output of both detector subunits are differentially weighted $(0.3: 1)$. Prior to this spatial summation the individual movement detector channels are shunted via presynaptic inhibition $(-)$ ) by a large-field "pool cell." The latter receives excitatory input (-4) from both movement 


\section{Discussion}

The fly visual system turned out to be an excellent model system for studying the processing of motion information (Reichardt and Poggio 1976; Poggio and Reichardt 1976; Buchner 1984; Reichardt 1986). It can be used as a model system in this regard, since there is now good evidence that movement detection is based on essentially the same principles in physiologically as distant species such as in man and the fly (e.g. van Doorn and Koenderink 1982a, b; van Santen and Sperling 1984; Baker and Braddick 1985; Wilson 1985). The evaluation of motion information is a purely local process. Each movement detector is assumed to consist of two mirror-symmetrical subunits which share the same two neighbouring retinal input channels. In the simplest version of the detector the signals of only one input channel are in some way delayed and then multiplied with the undelayed signals of the other input channel. The final movement detector response is obtained by subtracting the outputs of the two detector subunits (see Fig. 1). In the present study an algorithm has been developed which accounts for the dynamic response properties inherent in this type of movement detector. The significance of this algorithm could be experimentally established for the fly visual system. This was done by comparing the spatially integrated movement detector response with the functional properties of the directionally-selective motion-sensitive Horizontal Cells. These reside in the lobula plate, the posterior part of the third visual ganglion (see Fig. 4) and receive input from large parts of the visual field (Hausen 1982a, b). The algorithm for the movement detector response put forward here represents a generalization of the approach taken by Reichardt and Guo (1986). It represents a generalization in so far as it can be applied to a much wider class of moving stimuli. The only constraint imposed on the detector input functions is that they need to possess a convergent time-dependent Taylor series for any value of the spatial coordinate. This is not a too restrictive condition for most practical purposes, if one takes into account that the spatial frequency content of the incoming signal is confined in insects by the rather broad angular sensitivity functions of the photoreceptors (for review see Hardie 1985) and in vertebrates by the initial information processing stages (e.g. Enroth-Cugell and Robson 1966), respectively. This prevents the spatial derivatives in (8), (9), (11), and (13) from becoming too large. Moreover, under natural conditions retinal velocity changes and, consequently, the time derivatives of the function describing the displacement of the pattern are limited in size.

In the following, four major questions will be discussed which all shed some light on the range of applicability and the functional significance of the different approximations of the movement detector output and, in particular, the generalized theory presented here. $i$ ) Why are movement detectors of the type discussed here not velocity sensors? ii) What is the significance of spatial integration of the local movement detectors with respect to the representation of motion information? iii) How can the movement detector filter time constant be estimated? $i v)$ What are the functional consequences of the dynamic response properties of a spatially integrated movement detector array?

\subsection{Movement and Velocity Computation}

The motion detector of the type discussed here is no pure velocity sensor because its output is not unambiguously determined by the velocity of the stimulus pattern irrespective of the pattern's textural properties. This is known for long from the early studies on motion detection (Hassenstein and Reichardt 1956; Reichardt 1957, 1961; Reichardt and Varjú 1959; Varjú 1959; Götz 1964), but is also particularly obvious in the formulation of the movement detector

detector subunits. The following equation relates the output of the network $R(t)$ to its movement detector input $y\left(x_{i}, t\right)$

$R(t)=\sum_{i=1}^{N}\left[\frac{\left|y\left(x_{i}, t\right)\right|}{\beta+\left(\sum_{i=1}^{N}\left|y\left(x_{i}, t\right)\right|\right)^{q}}\right]^{n} \cdot \operatorname{sgn}\left[y\left(x_{i}, t\right)\right]$.

$N$ denotes the number of movement detectors in the array, $\beta$ the coefficient of shunting inhibition, $q<1$ approximates a saturation characteristic of the pool cell and $n$ represents the non-linearity in the synaptic transmission between the detector channels and the output cell of the network. To account for the characteristic spatial integration properties the model parameters have to be chosen appropriately $(\beta=0.001 ; n=1.25 ; q=0.5)$. As movement detector filter $F$ a low-pass of second order was used. Its impulse response function is shown in the inset. The oscillation amplitudes $A$, given in units of the spatial wavelength $\lambda$ of the pattern, were chosen as in the corresponding experiments (see Fig. 4). The ratio of the filter time constant $\tau$ and the temporal period $T$ was adjusted as to match the corresponding experimental results as closely as possible. At the bottom of each diagram the time-dependent deviation of the stimulus pattern from its mean position is shown. Downward deflections of the stimulus trace denote clockwise motion. On the basis of the characteristic time course of the computer simulated and the experimentally determined Horizontal Cell response the time constant of the movement detector filter can be estimated (for further details see text) 
theory as outlined here. In the power series representing the movement detector output [see (13)] each term consists of a time-dependent component, but also of a component which depends, in addition, in a non-linear way on the properties of the pattern. Even the exclusively time-dependent components of the movement detector response are not solely determined by the stimulus velocity, but rather by a weighted sum of velocity and all its higher order temporal derivatives [see (11)].

The experimental evidence in favour of this kind of motion detection scheme in both the insect as well as the human visual system is only indirect but, nevertheless, very specific. For methodological reasons, this evidence is not primarily due to electrophysiological recording from the local elements involved in the motion detection process. It has rather been obtained from representations of either the time averaged or the spatially integrated movement detector response as they manifest themselves at the behavioural or the neuronal level and, in case of man, in psychophysical experiments. Mainly two kinds of experimental evidence led to the conclusion that motion detection is not accomplished by pure velocity sensors. i) The amplitude of the time averaged as well as the spatially integrated movement detector response depends also on the textural properties of the stimulus patterns rather than on velocity alone. ii) Only within a limited dynamic range of pattern motion is the time course of the spatially integrated movement detector response proportional to pattern velocity.

The original evidence that the output of a movement detector is not unambiguously determined by the stimulus velocity has been derived from the optomotor turning response of insects on the basis of experiments where the stimulus consisted of grating patterns moving with a constant velocity. Under these conditions the time-averaged and spatially integrated movement detector response depends on the contrast frequency which is the ratio of the angular velocity of the pattern and its spatial wavelength, rather than on velocity itself (Kunze 1961; Götz 1964, 1972; McCann and MacGinitie 1965; Eckert 1973; Buchner 1984). This conclusion is, of course, consistent with the movement detector theory as formulated here for a much wider class of visual stimuli; this can easily be verified on the basis of (18). The same contrast frequency dependence as of the optomotor turning reaction has been found at the neuronal level in those large-field neurones of the fly's third visual ganglion which are involved in the control of this behavioural response component (Eckert 1980).

It is interesting to note, that under stationary stimulus conditions the output of the human motion detection system induced by grating patterns moving with constant velocity appears to be essentially determined by the contrast frequency (or, in the nomenclature used in the psychophysical literature, the temporal frequency) rather than by the pattern velocity itself. This holds true for the various psychophysical criteria which are used to study the human motion detection system (motion aftereffect: e.g. Pantle 1974; Wright and Johnston 1985; directionally selective adaptation: Tolhurst 1973; contrast sensitivity of moving gratings: Kelly 1979; contrast threshold of directional selectivity: Burr and Ross 1982; Anderson and Burr 1985; perceived velocity: Diener et al. 1976). Surprisingly, all these results have not been discussed with respect to the mechanism underlying the evaluation of motion information. In analogy to the insect data, however, they provide strong evidence that motion detection even in the human visual system is not accomplished by pure velocity sensors but by movement detectors which evaluate information on both motion as well as the textural properties of the stimulus pattern. This finding is, thus, in accordance with those psychophysical results which explicitly were interpreted to speak in favour of essentially the same movement detection scheme in the human and the insect visual system (e.g. van Doorn and Koenderink 1982a, b; van Santen and Sperling 1984; Wilson 1985). It should be emphasized that this conclusion is not affected by the evidence (e.g. Pantle et al. 1978; Anderson and Burr 1985) for spatial frequency band-pass filters in the movement detector input channels of man. This only means that motion detection operates independently in different spatial frequency bands.

The pattern effects discussed above do not only occur under stationary stimulus conditions. Only recently they have also been theoretically predicted and experimentally verified in the optomotor turning response of the fly to instationary pattern motion (Reichardt and Guo 1986). Although the response is about proportional to pattern velocity in the dynamic range studied by Reichardt and Guo (1986), its amplitude is determined by the textural properties of the pattern.

Another reason - not discussed so far in the literature - why a movement detector does not represent a pure velocity sensor has been analysed theoretically and experimentally in this paper. It can be derived from the spatially integrated response of an array of movement detectors. The time course of this response is proportional to pattern velocity only within a limited dynamic range of pattern motion, i.e. when the pattern velocity and its time derivatives are sufficiently small [see (14)]. The theoretical approach of Reichardt and Guo (1986), therefore, can only be applied within this range. Beyond this dynamic range qualitative deviations in the response profiles from being propor- 
tional to pattern velocity become obvious. This has been predicted in the present paper on the basis of the generalized time-dependent formulation of the motion detector output. If, for instance, the oscillation frequency and amplitude of a sinusoidally oscillated sine-wave grating are sufficiently small, the spatially integrated movement detector response is virtually a sinusoid and, thus, apart from a slight phase shift, proportional to pattern velocity (see e.g. Fig. 2 ; see also Thorson 1964). With increasing oscillation amplitude and/or frequency, however, the resulting increment in the response amplitude decreases until the response profiles flatten and the amplitude no longer increases. Eventually characteristic deflections become visible (see Fig. 2). These dynamic response properties of the spatially integrated response of an array of movement detectors have not only been predicted theoretically (see Sects. 3 and 4). In case of the fly they could also be confirmed experimentally in the response of the largefield Horizontal Cell (see Fig. 4) as well as at the behavioural level in the optomotor yaw torque response (Guo and Reichardt 1987). These experimental results are incompatible with the view that the movement detectors in the visual system of the fly are pure velocity sensors.

\subsection{Significance of Spatial Integration for the Representation of Motion Information}

The limitations of the individual elementary movement detectors with respect to a representation of motion information are immediately obvious from the theory outlined in this study. In general, the output of a single movement detetcor is not proportional to pattern velocity [see (13)]. This is even true in the first approximation of the detector theory (Reichardt and Guo 1986) where the pattern-independent term in the equation describing the movement detector output [see (14)] is proportional to velocity. Since, however, the pattern-dependent term depends also on the timedependent displacement function, the movement detector output is a complicated function of time. It can thus be concluded that with this motion detection mechanism it is impossible, apart from special stimulus conditions, to represent a signal in the output of a local motion detecting element that is proportional to stimulus velocity. There is cursory evidence that both in the fly (De Voe 1980) as well as in the vertebrate visual cortex (e.g. Holub and Morton-Gibson 1981) the response of local directionally-selective motionsensitive units to a sine-wave grating is modulated in a way as expected on the basis of the movement detector theory.

Spatial integration of local movement detector input over sufficiently large patches of the visual field is a means, most likely the simplest one, to obtain at least in a limited dynamic range a signal that is proportional to pattern velocity (Reichardt and Guo 1986). Even beyond this dynamic range spatial integration between appropriately chosen limits eliminates the timedependence of the pattern-dependent terms in the functional representation (13) of the movement detector output.

Spatial integration of motion information is of widespread relevance in both the insect and vertebrate visual system. The present study has exploited the methodological advantages provided by the wide-field movement-sensitive Horizontal Cells of the fly. They are involved in the control of the optomotor turning reaction (Hausen 1981; Hausen and Wehrhahn 1983; Wehrhahn 1985) and represent the site of convergence of the local retinotopic movement information. This kind of spatial integration, inevitably, has to take place somewhere in the visual pathway, since a single output variable, such as the yaw torque of the entire animal, is controlled by a multitude of retinotopic input channels. This argument might be generally true wherever compensatory movements of the eyes, the head or the entire animal are driven by visual motion information. In fact, integration of motion information over considerable parts of the visual field is accomplished in various vertebrate species by directionally-selective motion-sensitive neurones of the Accessory Optic System. This part of the visual system is assumed to be involved in the control of compensatory eye and head movements (for review see Simpson 1984) and, thus, appears to serve, at least partly, equivalent functions as the lobula plate of the fly. Moreover, directionallyselective motion-sensitive cells with relatively large receptive fields are characteristic for the superior temporal sulcus of the monkey (area MT/V5; e.g. Zeki 1974). Whatever their functional significance might be they obviously integrate motion information within the confines of their receptive fields. In psychophysical experiments spatial integration has been found to play also a decisive role in human motion perception (e.g. Lappin and Bell 1976; Chang and Julesz 1983; van Doorn and Koenderink 1984; van Doorn et al. 1985).

It should be emplaasized that the term "integration" has been used in this section in a colloquial sense. It has not been intended to imply that the local motion information is integrated in all the examples mentioned above in a mathematical sense as has been done for simplicity in the theoretical predictions of Sect. 4. Instead, there is good evidence - to mention only the example analysed in this study - that the Horizontal Cells do not summate linearly their retinotopically organized movement detector input (Hausen 1982b). They rather show characteristic nonlinear spatial integration properties which have been 
interpreted in the context of figure-ground discrimination as evidence for a specific non-linear transformation of the movement detector output prior to spatial summation (Reichardt et al. 1983; Egelhaaf 1985a). In the present study the Horizontal Cell was simulated on the basis of this model (see Fig. 5). It is hard to calculate analytically in what way these transformations affect the time course of the Horizontal Cell response. A comparison of the spatially integrated movement detector output with and without this non-linear transformation, however, has been done by computer simulation. For the stimulus conditions tested and, in particular, for those employed in the experimental part of this study the time course of the response turned out to be virtually the same in both cases. Nevertheless, very small but systematic deviations can be found. This, however, is beyond the scope of this paper and will be discussed in more detail elsewhere (Reichardt and Egelhaaf in preparation). Hence, with respect to their dynamic response properties the Horizontal Cells appear to be sufficiently mimicked by linearly integrating their retinotopic movement-sensitive input. Whether this is true for the other systems cited above needs to be worked out experimentally as well as theoretically. In any case, however, some form of spatial integration is a necessary precondition for representing, at least in a certain dynamic range, a signal which is proportional to the velocity of the stimulus pattern.

\subsection{Estimation of the Filter Time Constant}

The dynamic properties of a movement detector are essentially determined by its filter time constant. This is particularly true for the dynamic range of stimulus motion where the integrated response of an array of movement detectors is proportional to the velocity of the stimulus pattern. The characteristic deviations of the integrated response from being proportional to pattern velocity which occur beyond this dynamic range can thus be exploited to estimate the time constant of the movement detector filter. This has been done for the movement detectors in the input circuitry of the wide-field Horizontal Cells of the fly third optical ganglion. The parameters of the stimuli and the resulting cellular responses were related to the corresponding model simulations of the Horizontal Cell response (see inset of Fig. 5; Reichardt et al. 1983; Egelhaaf 1985a). In this way, the movement detector time constant has been estimated to lie within the range between $5 \mathrm{~ms}$ and $10 \mathrm{~ms}$. This is approximately the same range as has recently been derived on the basis of behavioural data (Guo and Reichardt 1987).

How critical are the specific assumptions made in the model simulations with respect to the numerical value of the time constant? In the computer simulations of the Horizontal Cell response a low-pass of second order was chosen as motion detector filter. This choice represents a good compromise between a qualitatively good model fit and an as simple model as possible. As is argued in the result section, the numerical value of the movement detector time constant determined for the fly's visual system does not depend much on the choice of the filter. By far the largest values were obtained with a pure delay. This, however, is likely to be an unrealistic assumption suggesting that the values obtained with this kind of filter might be most probably an overestimation of the true value.

It was not necessary in the computer simulations of the Horizontal Cell response to make allowance for the processing of the incoming signals distally to the movement detectors in order to reach a good agreement with the characteristic time course of the cellular response. This suggests that the time course of the Horizontal Cell response is essentially determined by the specific functional characteristics of the movement detector itself rather than by more peripheral filters. It is beyond the scope of this paper to analyse in what way the characteristic features of the movement detector response are affected by temporally prefiltering the detector input. Only one point should be mentioned here. The kind of peripheral filtering and the corresponding filter time constants are, in principle, not very critical, if only the response under stationary conditions is taken into account. This is, however, radically different, if, as in the present study, dynamic stimulus conditions are concerned. As can be shown by computer simulations, the detector input function does not allow much prefiltering for the movement detector output signal still to be compatible with the timedependent cellular response. This might be surprising at first sight, since there is ample evidence on the basis of single cell recording for an extensive transformation of the retinal light intensity distribution in the peripheral visual system of the fly (for review see e.g. Laughlin 1981). What role these cellular elements play in the context of movement detection, however, has by no means been settled so far. In any case, it should be emphasized that it needs first to be shown whether the temporal filter properties of a peripheral neuronal element can be reconciled with our detailed knowledge on the motion detection mechanism and the experimental results on its characteristic output properties, before this cell can be accepted as an input element to the motion detection system.

In two recent papers on the H1-neurone, another lobula plate large-field tangential cell of the fly, the response to brief jumps of the stimulus pattern in the cell's preferred direction has been found to narrow considerably, if the animal was preexposed to large- 
field motion (Maddess and Laughlin 1985; de Ruyter van Steveninck 1986; de Ruyter van Steveninck et al. 1986). This change in the time course of the response has been interpreted as an adaptation of the movement detector time constant (de Ruyter van Steveninck 1986; de Ruyter van Steveninck et al. 1986). This kind of adaptation of the time constant can neither be confirmed nor excluded on the basis of the present study. It cannot be confirmed, since the time course of the Horizontal Cell response could, in principle, be simulated for all stimulus conditions used in the experiments by a single value of the time constant (see Sect. 6). On the other hand, an adaptation of the time constant cannot be excluded, since it can be determined in the way as was done here only on the basis of the characteristic deformations of the response profile; these, however, can only be observed, if the stimulus velocity and its higher order time derivatives are sufficiently large. No definite value for the time constant can be calculated, of course, as long as the response is approximately proportional to the stimulus velocity. Under these conditions only the largest time constant can be determined that is compatible with the experimental data. The very short time constant estimated here thus characterizes the performance of the motion detection system at its maximum capacity to resolve the rate at which velocity changes. Although the interpretations of de Ruyter van Steveninck et al. (1986) are highly questionable (Borst and Egelhaaf 1987), it is interesting to note that time constants of $5 \mathrm{~ms}-10 \mathrm{~ms}$ as determined by our technique correspond to the smallest time constants they obtain after high-velocity adaptation of the cell.

To our knowledge, no other estimates of the movement detector time constant in the fly are available so far apart from the already mentioned study of de Ruyter van Steveninck et al. (1986). This is surprising, since, in principle time constants could have been derived on the basis of the contrast frequency optima of either the optomotor turning reaction (Götz 1964; McCann and MacGinitie 1965; Eckert 1973; Buchner 1984; Wehrhahn 1985; Borst and Bahde 1987) or the response of lobula plate large-field tangential neurones which are involved in the control of this behavioural response component (Eckert 1980; Mastebroek et al. 1980; Hausen 1982b; Maddess and Laughlin 1985). In all these studies the contrast frequency optima lie somewhere within the range between 1 and $10 \mathrm{~Hz}$ depending on the species and the exact stimulus conditions. On the basis of the detector model used here this range would correspond to time constants between about $90 \mathrm{~ms}$ and $9 \mathrm{~ms}$. It should be noted, however, that this kind of estimation of the time constant is rather unreliable, since the contrast frequency curves are very flat around their optima. The present method to estimate the time constant appears to be much more sensitive, since a tenfold change in frequency in the range of maximal response amplitudes leads to dramatic qualitative differences in the response profiles (see Figs. 2, 4, and 5).

In human motion perception the estimated movement detector time constants range between about $80 \mathrm{~ms}$ and $13 \mathrm{~ms}$ (e.g. Schouten 1967; van Doorn and Koenderink 1982a; Baker and Braddick 1985; Koenderink et al. 1985; Wilson 1985). This variability is not much surprising, if one takes into account that these estimates were obtained with very different techniques and psychophysical criteria for movement detection. It is, however, interesting to note that these values for the time constant fall, roughly speaking, in the same range as the estimates obtained for the fly motion detection system. The likely functional consequences of this will be discussed in the next section.

\subsection{Functional Consequences of the Dynamic Response Properties of a Spatially Integrated Movement Detector Array}

The characteristic deviations of the spatially integrated response of an array of movement detectors from being proportional to the velocity of the stimulus pattern are not the result of extreme stimulus conditions far beyond the optimal operating range of the system. They have rather been predicted to occur while the spatially integrated movement detector response shows its maximal response amplitudes (see Fig. 2). This prediction could be experimentally confirmed for the fly Horizontal Cells (see Fig. 4). The question, therefore, arises whether any functional significance can be attributed to these stimulus dependent deformations of the response profile or whether they merely represent an unintended by-product of the motion detection mechanism. This question cannot be answered in general, but only if the computational tasks of the particular systems are known. In case of the Horizontal Cells in flies the latter alternative appears to us to be the more likely one. For the sake of argument, it will, therefore, be assumed in the further considerations that the actual task of a motion detection system is to convey a signal which is proportional to pattern velocity.

As has been shown in the present study, this task can only be accomplished, if the movement detector time constant, the spatial frequency content of the stimulus pattern and the dynamic range of motion are properly matched to each other. The dynamic range of stimulus motion which leads to a spatially integrated movement detector response that is proportional to pattern velocity can be extended in two ways towards larger velocities and velocity changes. Either the time 
constant of the movement detector has to be reduced or, for a given time constant, the high spatial frequency components of the pattern have to be prevented from contributing to the movement detector response. The latter possibility can be achieved by low-pass filtering the incoming signal. In insects spatial low-pass filtering is already achieved in the retina by convolving the retinal image of the visual scene with the relatively broad angular sensitivity distribution of the photoreceptors (for review see Hardie 1985; see also Götz 1965). By increasing the width of the angular sensitivity distribution the cut-off frequency of the spatial lowpass filter and, concomitantly, the spatial resolution of the eye decreases. As a consequence, larger velocities and velocity changes can be represented in the spatially integrated movement detector response without distortion. For a given movement detector time constant this implies a trade-off between the spatial resolution of the eye and the dynamic range of pattern motion which at the output of the motion detection system results in a signal that is about proportional to pattern velocity. As a consequence, this dynamic range should be considerably smaller in human than in insect motion perception, at least for the psychophysically determined high spatial frequency channels, since the estimated filter time constants are in the same order of magnitude in both cases (see Sect. 7.3). This prediction derived from the movement detector theory developed here represents another challenge to the conclusion that movement detection in the fly and human visual system is based on essentially the same principles.

Acknowledgements. We are grateful to Dr. A. Borst for his help in doing the calculations of the contour plot of Fig. 3. Without his guidance in using the ASYST-software, the computer programming would have taken us much more time. Dr. A. Borst, Prof. K. G. Götz, Dr. K. Hausen, and Dr. C. Wehrhahn are all thanked for reading and criticizing the manuscript. The help of K. Bierig and G. Dechert in preparation of the figures and the secretarial assistance of I. Geiss are gratefully acknowledged.

\section{References}

Adelson EH, Bergen JR (1985) Spatiotemporal energy models for the perception of motion. J Opt Soc Am A 2:284-299

Anderson SJ, Burr DC (1985) Spatial and temporal selectivity of the human motion detection system. Vision Res 8:1147-1154

Baker LB, Braddick OJ (1985) Temporal properties of the shortrange process in apparent motion. Perception 14:181-192

Borst A, Bahde S (1987) Comparison between the movement detection systems underlying the optomotor and the landing response in the housefly. Biol Cybern (in press)

Borst A, Egelhaaf M (1987) Temporal modulation of luminance adapts time constant of fly movement detectors. Biol Cybern (in press)

Buchner E (1984) Behavioural analysis of spatial vision in insects. In: Ali MA (ed) Photoreception and vision in invertebrates. Plenum Press, New York London, pp 561-621
Burr DC, Ross J (1982) Contrast sensitivity at high velocities. Vision Res 22:479-484

Chang JJ, Julesz B (1983) Displacement limits, directional anisotropy and direction versus form discrimination in random-dot cinematograms. Vision Res 23:639-646

De Voe RD (1980) Movement sensitivities of cells in the fly's medulla. J Comp Physiol 138:93-119

Diener HC, Wist ER, Dichgans J, Brandt T (1976) The spatial frequency effect on perceived velocity. Vision Res 16:169-176

van Doorn AJ, Koenderink JJ (1982a) Temporal properties of the visual detectability of moving spatial white noise. Exp Brain Res 45:179-188

van Doorn AJ, Koenderink JJ (1982b) Spatial properties of the visual detectability of moving white noise. Exp Brain Res 45:189-195

van Doorn AJ, Koenderink JJ (1984) Spatiotemporal integration in the detection of coherent motion. Vision Res $24: 47-53$

van Doorn AJ, Koenderink JJ, van de Grind WA (1985) Perception of movement and correlation in stroboscopically presented noise patterns. Perception 14:209-224

Eckert H (1973) Optomotorische Untersuchungen am visuellen System der Stubenfliege Musca domestica L. Kybernetik 14:1-23

Eckert H (1980) Functional properties of the H1-neurone in the third optic ganglion of the blowfly, Phaenicia. J Comp Physiol 135:29-39

Egelhaaf M (1985a) On the neuronal basis of figure-ground discrimination by relative motion in the visual system of the fly. I. Behavioural constraints imposed on the neuronal network and the role of the optomotor system. Biol Cybern $52: 123-140$

Egelhaaf M (1985b) On the neuronal basis of figure-ground discrimination by relative motion in the visual system of the fly. II. Figure-detection cells, a new class of visual interneurones. Biol Cybern 52:195-209

Egelhaaf M (1985c) On the neuronal basis of figure-ground discrimination by relative motion in the visual system of the fly. III. Possible input circuitries and behavioural significance of the FD-cells. Biol Cybern 52:267-280

Enroth-Cugell C, Robson JG (1966) The contrast sensitivity of retinal ganglion cells of the cat. J Physiol 187:517-552

Götz KG (1964) Optomotorische Untersuchung des visuellen Systems einiger Augenmutanten der Fruchtfliege Drosophila. Kybernetik 2:77-92

Götz KG (1965) Die optischen Übertragungseigenschaften der Komplexaugen von Drosophila. Kybernetik 2:215-221

Götz KG (1972) Principles of optomotor reactions in insects. Bibl Ophthalmol 82:251-259

Gradshteyn IS, Ryzhik IW (1965) Table of integrals, series, and products. Academic Press, New York San Francisco London

Guo A, Reichardt W (1987) An estimation of the time constant of movement detectors. Naturwissenschaften (in press)

Hardie RC (1985) Functional organization of the fly retina. In: Autrum H, Ottoson D, Perl ER, Schmidt RF, Shimazu H, Willis WD (eds) Progress in sensory physiology 5. Springer, Berlin Heidelberg New York, pp 1-79

Hassenstein B, Reichardt W (1956) Systemtheoretische Analyse der Zeit-, Reihenfolgen- und Vorzeichenauswertung bei der Bewegungsperzeption des Rüsselkäfers Chlorophanus. Z Naturforsch 11b:513-524

Hausen K (1981) Monocular and binocular computation of motion in the lobula plate of the fly. Verh Dtsch Zool Ges 74:49-70 
Hausen K (1982a) Motion sensitive interneurons in the optomotor system of the fly. I. The Horizontal Cells: structure and signals. Biol Cybern 45:143-156

Hausen K (1982b) Motion sensitive interneurons in the optomotor system of the fly. II. The Horizontal Cells: receptive field organization and response characteristics. Biol Cybern 46:67-79

Hausen K, Wehrhahn C (1983) Microsurgical lesion of horizontal cells changes optomotor yaw responses in the blowfly Calliphora erythrocephala. Proc $\mathbf{R}$ Soc London B 219:211-216

Hengstenberg R (1982) Common visual response properties of giant vertical cells in the lobula plate of the blowfly Calliphora. J Comp Physiol 149:179-193

Holub RA, Morton-Gibson M (1981) Response of visual cortical neurons of the cat to moving sinusoidal gratings: responsecontrast functions and spatiotemporal interactions. J Neurophysiol 46:1244-1259

Kelly DH (1979) Motion and vision. II. Stabilized spatiotemporal threshold surface. J Opt Soc Am 69:1340-1349

Koenderink JJ, van Doorn AJ, van de Grind WA (1985) Spatial and temporal parameters of motion detection in the peripheral visual field. J Opt Soc Am A 2:252-259

Kunze $P$ (1961) Untersuchung des Bewegungssehens fixiert fliegender Bienen. Z Vergl Physiol 44:656-684

Lappin JS, Bell HH (1976) The detection of coherence in moving random-dot patterns. Vision Res 16:161-168

Laughlin SB (1981) Neural principles in the peripheral visual systems of invertebrates. In: Autrum $\mathrm{H}$ (ed) Handbook of sensory physiology VII/6B. Springer, Berlin Heidelberg New York, pp 133-280

Maddess T, Laughlin SB (1985) Adaptation of the motionsensitive neuron $\mathrm{H} 1$ is generated locally and governed by contrast frequency. Proc R Soc London 225:251-275

Mastebroek HAK, Zaagman WH, Lenting BPM (1980) Movement detection: Performance of a wide-field element in the visual system of the blowfly. Vision Res 20:467-474

McCann GD, MacGinitie GF (1965) Optomotor response studies of insect vision. Proc R Soc London 163:369-401

Pantle A (1974) Motion aftereffect magnitude as a measure of the spatio-temporal response properties of direction-sensitive analyzer. Vision Res 14:1229-1236

Pantle A, Lehmkuhle S, Candill M (1978) On the capacity of directionally selective mechanisms to encode different dimensions of moving stimuli. Perception 7:261-267

Poggio T, Reichardt W (1973) Considerations on models of movement detection. Kybernetik 13:223-227

Poggio T, Reichardt W (1976) Visual control of orientation behaviour in the fly. Part II. Towards the underlying neural interactions. Q Rev Biophys 9:377-438

Reichardt W (1957) Autokorrelations-Auswertung als Funktionsprinzip des Zentralnervensystems (bei der optischen Wahrnehmung eines Insektes). Z Naturforsch 12b:448-457

Reichardt W (1961) Autocorrelation, a principle for evaluation of sensory information by the central nervous system. In: Rosenblith WA (ed) Principles of sensory communication. Wiley, New York, pp 303-317

Reichardt W (1985) Computation of sensory information by the visual system of the fly (from behaviour to neuronal circuitry). In: Haken $H$ (ed) Complex systems - operational approaches in neurobiology, physics, and computers. Springer, Berlin Heidelberg New York Tokyo, pp 38-57
Reichardt W (1986) Processing of optical information by the visual system of the fly. Vision Res 26:113-126

Reichardt W, Guo A (1986) Elementary pattern discrimination (behavioural experiments with the fly Musca domestica). Biol Cybern 53:285-306

Reichardt W, Poggio T (1976) Visual control of orientation behaviour in the fly. Part I. A quantitative analysis. Q Rev Biophys 9:311-375

Reichardt W, Varjú D (1959) Übertragungseigenschaften im Auswertesystem für das Bewegungssehen (Folgerungen aus Experimenten an dem Rüsselkäfer Chlorophanus viridis). Z Naturforsch 14b:674-689

Reichardt W, Poggio T, Hausen K (1983) Figure-ground discrimination by relative movement in the visual system of the fly. Part II. Towards the neural circuitry. Biol Cybern (Suppl) 46:1-30

de Ruyter van Steveninck RR (1986) Real-time performance of a movement-sensitive neuron in the blowfly visual system. Doctoral Dissertation, Rijksuniversiteit de Groningen

de Ruyter van Steveninck RR, Zaagman WH, Mastebroek HAK (1986) Adaptation of transient responses of a movementsensitive neuron in the visual system of the blowfly Calliphora erythrocephala. Biol Cybern 54:223-236

van Santen JPH, Sperling G (1984) Temporal covariance model of human motion perception. J Opt Soc Am A 1:451-473

van Santen JPH, Sperling G (1985) Elaborated Reichardt detectors. J Opt Am A 2:300-321

Schouten IF (1967) Subjective stroboscopy and a model of visual movement detectors. In: Wathen-Dum W (ed) Models for the perception of speech and visual form. MIT Press, Cambridge MA, pp 4455

Simpson JI (1984) The accessory optic system. Ann Rev Neurosci 7:13-41

Thorson J (1964) Dynamics of motion perception in the desert locust. Science 149:69-71

Tolhurst DJ (1973) Separate channels for the analysis of the shape and the movenent of a moving visual stimulus. $J$ Physiol 231:385 402

Varjú D (1959) Optomotorische Reaktionen auf die Bewegung periodischer Helligkeitsmuster (Anwendung der Systemtheorie auf Experimente am Rüsselkäfer Chlorophanus viridis). Z Naturforsch 14b:724-735

Wehrhahn C (1985) Visual guidance during flight. In: Kerkut G, Gilbert L (eds) Comprehensive insect physiology, biochemistry and pharmacology. Pergamon Press, Oxford, pp 673-683

Wilson HR (1985) A model for direction selectivity in threshold motion perception. Biol Cybern 51:213-222

Wright MJ, Johnston A (1985) Invariant tuning of motion aftereffect. Vision Res 25:1947-1955

Zeki SM (1974) Functional organization of a visual area in the posterior bank of the superior temporal sulcus of the monkey. J Physiol 236:549-573

Received: December 1, 1986

Dr. Martin Egelhaaf

Max-Planck-Institut

für Biologische Kybernetik.

Spemannstrasse 38

D-7400 Tübingen

Federal Republic of Germany 